\title{
Trabajo en equipo y planificación estratégica en la Facultad de Ciencias Empresariales de la Universidad José Faustino Sánchez Carrión - 2019
}

\author{
Teamwork and strategic planning in the Faculty of Business Sciences of the José Faustino \\ Sánchez Carrión University -2019
}

M(o) Pedro Javier Yovera Rodríguez'

\section{RESUMEN}

La investigación se llevó acabo en la Facultad de Ciencias Empresariales, el cual se dedica a instruir estudiantes líderes que posean competencias y habilidades para la investigación, la innovación y gestión; con valores y sentido humanístico; con la capacidad de aportar para el crecimiento del país, a la protección y cuidado del medio ambiente, de igual forma a la consolidación de la identidad local y nacional. La presente investigación posee un enfoque cuantitativo, de tipo aplicada y nivel correlacional. Además, es de diseño no experimental transversal. El principal objetivo es definir la relación existente entre el trabajo en equipo y la planificación estratégica en la Facultad de Ciencias Empresariales, de la Universidad José Faustino Sánchez Carrión, 2019. Para el procesamiento de datos se empleó una encuesta sobre el trabajo en equipo y planificación estratégica a 86 personas entre docentes y trabajadores administrativos. En cuanto al instrumento de recolección de datos, se ejecutó un cuestionario acerca de las variables en estudio contando con un total de 29 ítems aplicadas a una Escala de Likert. De igual forma la validez y la confiabilidad del instrumento fueron efectuadas según el coeficiente de Alfa de Cronbach y los resultados que se obtuvieron fueron 0.89 , por tanto, el instrumento es confiable y consistente. Para establecer la relación existente entre las dos variables se empleó, el estadístico no paramétrica Rho de Spearman. Lográndose demostrar que existe relación entre el trabajo en equipo y la planificación estratégica en la Facultad de Ciencias Empresariales, de la Universidad José Faustino Sánchez Carrión, 2019. Además, la correlación de Rho de Spearman es 0.605 , de acuerdo a la escala de Bisquerra dicha correlación es positiva y moderada.

Palabras clave: Trabajo en equipo y Planificación estratégica

\section{ABSTRACT}

The research was carried out in the Faculty of Business Sciences, which is dedicated to instruct leading students who possess competences and abilities for research, innovation and management; with values and humanistic sense; with the ability to contribute to the development of the country, to the protection and care of the environment, as well as to the affirmation of our local and national identity. The present investigation is of quantitative approach, of applied type and correlational level. In addition, it is of non-experimental transversal design. The main objective is to define the relationship that exists between teamwork and strategic planning in the Faculty of Business Studies, Universidad José Faustino Sánchez Carrión, 2019. For data processing, an opinion poll on teamwork and strategic planning was applied to 86 people among teachers and administrative workers. Regarding the data collection instrument, a questionnaire was applied about the two variables with a total of 29 items with a Likert scale. Likewise, the validity and reliability of the instrument were made according to the Cronbach's Alpha coefficient and the results obtained were 0.89 , therefore, the instrument is reliable and consistent. To establish the existing relationship between the two variables, we applied the Spearman nonparametric Rho statistic. Being able to demonstrate that there is a relationship between teamwork and strategic planning in the Faculty of Business Studies, José Faustino Sánchez Carrión University, 2019. In addition, Spearman's Rho correlation is 0.605 , according to the scale of Bisquerra said Correlation is positive and moderate.

Keywords: Teamwork and Strategic Planning.

\section{NTRODUCCIÓN}

La presente tesis denominada "El Trabajo en Equipo y Planificación estratégica en la Facultad de Ciencias Empresariales de la Universidad Nacional José Faustino Sánchez Carrión", tiene como objetivo determinar la relación que existe entre el trabajo en equipo y la planificación estratégica en la Facultad de Ciencias Empresariales, de la Universidad José Faustino Sánchez Carrión, 2019; es decir, consiste en investigar la dinámica de equipos, el liderazgo, la comunicación y la motivación en dicha institución. También se investigó la planificación estratégica en la Facultad de Ciencias Empresariales.

Recibido 15/10/2020 Aprobado 04/01/2021

Este es un artículo de acceso abierto, distribuido bajo los términos de la Licencia Creative Commons Atribución 4.0 Internacional (http://creativecommons.org/licenses/by/4.0/)

\section{(c) (i)}

'M(o) de la Facultad de Ciencias Empresariales de La Universidad Nacional José Faustino Sánchez Carrión. Huacho. Huaura. Lima. Perú. ORCID: https://orcid.org/ 0000-0002-2365-0175. Pertenece al Colegio de Administradores de Lima. Email: pyovera@unjfsc.edu.pe 
Sin embargo, las recomendaciones que se sugieren en esta investigación permitirán continuar mejorando el trabajo en equipo y la planificación estratégica.

La presente investigación está organizada en seis capítulos de la siguiente manera:

Capítulo I: planteamiento del problema, abarca la explicación de la realidad problemática, formulación del problema y objetivos de la investigación general y especifico.

Capitulo II: Marco Teórico, establece los antecedentes de la investigación, definición de conceptos, bases teóricas, hipó tesis general y especifico.

Capitulo III: Contiene la metodología, el cual comprende el diseño metodológico, enfoque y tipo de investigación, población y muestra, técnica de recolección de datos y procesamiento de la información.

Capitulo IV: Contiene los resultados de la encuesta en tablas, figuras e interpretaciones.

Finalmente.

Capítulo V: Contiene la discusión.

Capítulo VI: Contiene la conclusión y recomendaciones de la presente investigación.

\section{Problema general}

¿Cuál es la relación existe entre el trabajo en equipo y la planificación estratégica en la Facultad de Ciencias Empresariales, de la Universidad José Faustino Sánchez Carrión, 2019?

\section{Problemas específicos}

¿Qué asociación existe la dinámica de equipos y la planificación estratégica en la Facultad de Ciencias Empresariales, de la Universidad José Faustino Sánchez Carrión, 2019?

¿Qué relación existe entre el liderazgo y la planificación estratégica en la Facultad de Ciencias Empresariales, de la Universidad José Faustino Sánchez Carrión, 2019?

¿Cuál es la relación que existe entre la comunicación y la planificación estratégica en la Facultad de Ciencias Empresariales, de la Universidad José Faustino Sánchez Carrión, 2019?

¿Cómo se relaciona la motivación y planificación estratégica en la Facultad de Ciencias Empresariales, de la Universidad José Faustino Sánchez Carrión, 2019?

\section{OBJETIVO}

Determinar la relación que existe entre el trabajo en equipo y la planificación estratégica en la Facultad de Ciencias Empresariales, de la Universidad José Faustino Sánchez Carrión, 2019.

\section{Objetivos específicos}

Determinar la asociación entre la dinámica de equipos y la planificación estratégica en la Facultad de Ciencias Empresariales, de la Universidad José Faustino Sánchez Carrión, 2019.

Determinar la relación que existe entre el liderazgo y la planificación estratégica en la Facultad de Ciencias Empresariales, de la Universidad José Faustino Sánchez Carrión, 2019.

Determinar la relación que existe entre comunicación y planificación estratégica en la Facultad de Ciencias Empresariales, de la Universidad José Faustino Sánchez Carrión, 2019.

Determinar cómo se relaciona la motivación y la planificación estratégica en la Facultad de Ciencias Empresariales, de la Universidad José Faustino Sánchez Carrión, 2019.

\section{MÉTODOS Y MATERIALES}

\section{Nivel de Investigación}

La presente investigación es de tipo descriptivo correlacional, porque trata de responder a un problema de corte teórico y tiene por finalidad describir un fenómeno o una situación mediante el estudio del mismo en una circunstancia temporal-espacial determinada.

Según Arias, (2012) la finalidad de la investigación correlacional es determinar el grado de relación o asociación (no causal) existente entre dos o más variables. En estos estudios, primero se miden las variables y luego, mediante pruebas de hipótesis correlacionales y la aplicación de técnicas estadísticas, se estima la correlación. Aunque la investigación correlacional no establece de forma directa relaciones causales, puede aportar indicios sobre las posibles causas de un fenómeno. (p. 25)

La utilidad y el propósito principal de los estudios correlacionales es saber cómo se puede comportar un concepto o variable conociendo el comportamiento de otras variables relacionadas. Es decir, intentar predecir el valor aproximado que tendrá una variable en un grupo de individuos, a partir del valor obtenido en la variable o variables relacionadas. (Hernández, Fernández y Baptista, 2010, p.82).

La presente investigación es correlacional, porque trata de responder a un problema de corte teórico y tiene por finalidad determinar el grado de relación entre Trabajo en Equipo y Planificación Estratégica mediante el estudio del mismo en una circunstancia temporal espacial determinada.

\section{Tipo de Investigación}

La investigación realizada se caracteriza por ser de tipo aplicada, porque busca la generación de nuevo conocimiento y se fundamenta en los hallazgos 
tecnológicos de la investigación básica.

\section{Método}

La metodología que se utilizará para el proyecto es el método científico (hipotético-deductivo), ya que se inicia con la identificación y formulación del problema, se continúa con la formulación de los objetivos y las hipótesis; luego se elabora y aplica los instrumentos de investigación para obtener los datos y procesar los mismos para realizar la inferencia de la investigación.

\section{Población y muestra}

La población de la presente investigación está determinada por 86 trabajadores entre docentes y administrativos de la Facultad de Ciencias Empresariales.

La muestra se considera a la totalidad de la población en estudio.

\section{Técnicas de recolección de datos}

- Para la aplicación del instrumento se coordinó con el Decano de la Facultad de Ciencias Empresariales, para que a su vez solicite el permiso a los directores de cada escuela profesional y poder aplicar la encuesta a los docentes y trabajadores administrativos.

- Los cuestionarios fueron aplicados personalmente a los integrantes que conforman la población muestral de mi investigación. La aplicación de la encuesta será en una sesión de 30 minutos como máximo.

- La encuesta se aplicó dentro de las instalaciones de la Facultad de Ciencias Empresariales, la cual se inició dándoles las instrucciones, solicitándoles que respondan a todo el cuestionario. Para evitar el sesgo y se enfatizó en que no hay respuestas incorrectas.

- Cuando los encuestados culminen la encuesta se les fue revisando que todo el Ítems haya sido contestado.

\section{RESULTADOS}

\section{HIPÓTESIS GENERAL}

$\mathrm{H}_{0}$ : No existe relación entre el trabajo en equipo y la planificación estratégica en la Facultad de Ciencias Empresariales, de la Universidad José Faustino Sánchez Carrión, 2019.

$\mathrm{H}_{1}$ : Existe relación entre el trabajo en equipo y la planificación estratégica en la Facultad de Ciencias Empresariales, de la Universidad José Faustino Sánchez Carrión, 2019.

\section{DEMOSTRACIÓNDELAHIPÓTESIS}

Utilizamos el siguiente criterio: Si la significancia asintótica $(p)>$ al nivel de significancia $(0,05)$, se acepta la $\mathrm{H}_{0}$. Si el valor de $\mathrm{p}<0,05$ se rechaza $\mathrm{H}_{0}$.

Aplicamos SPSS v25:

\section{Tabla 1.}

Correlación entre el trabajo en equipo y la planificación estratégica

\begin{tabular}{|c|c|c|c|c|}
\hline & & & $\begin{array}{l}\text { Planificacción } \\
\text { estratégica }\end{array}$ & $\begin{array}{l}\text { Trabajo en } \\
\text { equipo }\end{array}$ \\
\hline \multirow{6}{*}{$\begin{array}{l}\text { Rho de } \\
\text { Spearman }\end{array}$} & & $\begin{array}{l}\text { Coeficiente de } \\
\text { correlación }\end{array}$ & 1,000 & $0,605^{* *}$ \\
\hline & $\begin{array}{l}\text { Planificación } \\
\text { estratégica }\end{array}$ & Sig. (bilateral) & & 0,000 \\
\hline & & $\mathrm{N}$ & 86 & 86 \\
\hline & \multirow{3}{*}{$\begin{array}{l}\text { Trabajo en } \\
\text { equipo }\end{array}$} & $\begin{array}{l}\text { Coeficiente de } \\
\text { correlación }\end{array}$ & $0,605^{* *}$ & 1,000 \\
\hline & & Sig. (bilateral) & 0,000 & \\
\hline & & $\mathrm{N}$ & 86 & 86 \\
\hline
\end{tabular}

La correlación es significativa en el nivel 0,01 (Bilateral)

\section{nterpretación:}

Como se observa en la tabla 1 la significancia asintótica $(0,000)$ es menor que el nivel de significación $(0,05)$, se rechaza la hipótesis nula y se acepta la hipótesis alterna (hipótesis del investigador). Es decir, existe relación entre el trabajo en equipo y la planificación estratégica en la Facultad de Ciencias Empresariales, de la Universidad José Faustino Sánchez Carrión, 2019. Además, la correlación de Rho de Spearman es 0,605, de acuerdo a la escala de Bisquerra dicha correlación es positiva y moderada.

\section{HIPÓTESIS ESPECIFICA 1}

$\mathrm{H}_{0}$ : No existe asociación entre la dinámica de equipos y la planificación estratégica en la Facultad de Ciencias Empresariales, de la Universidad José Faustino Sánchez 


\section{Carrión, 2019}

$\mathrm{H}_{1}$ : Existe asociación entre la dinámica de equipos y la planificación estratégica en la Facultad de Ciencias Empresariales, de la Universidad José Faustino Sánchez Carrión, 2019.

Tabla 2.

Asociación entre la dinámica de equipos y la planificación estratégica

\begin{tabular}{|c|c|c|c|c|}
\hline & & & $\begin{array}{l}\text { Dinámica de } \\
\text { equipos }\end{array}$ & $\begin{array}{l}\text { Planificacción } \\
\text { estratégica }\end{array}$ \\
\hline \multirow{6}{*}{$\begin{array}{l}\text { Rho de } \\
\text { Spearman }\end{array}$} & \multirow{3}{*}{$\begin{array}{l}\text { Dinámica de } \\
\text { equipos }\end{array}$} & $\begin{array}{l}\text { Coeficiente de } \\
\text { correlación }\end{array}$ & 1,000 & $0,393^{* *}$ \\
\hline & & Sig. (bilateral) & & 0,000 \\
\hline & & $\mathrm{N}$ & 86 & 86 \\
\hline & \multirow{3}{*}{$\begin{array}{l}\text { Planificación } \\
\text { estrátegica }\end{array}$} & $\begin{array}{l}\text { Coeficiente de } \\
\text { correlación }\end{array}$ & $0,393^{* *}$ & 1,000 \\
\hline & & Sig. (bilateral) & 0,000 & \\
\hline & & $\mathrm{N}$ & 86 & 86 \\
\hline
\end{tabular}

La correlación es significativa en el nivel 0,01 (Bilateral)

\section{Interpretación:}

En la tabla 13 se aprecia la significancia asintótica $(0,000)$ que es menor que el nivel de significación 0,05 , se rechaza la hipótesis nula y se acepta la hipótesis alterna (hipótesis del investigador). Es decir, existe asociación entre la dinámica de equipos y la planificación estratégica en la Facultad de Ciencias Empresariales, de la Universidad José Faustino Sánchez Carrión, 2019. Además, la correlación de Rho de Spearman es 0,393, de acuerdo a la escala de Bisquerra dicha correlación es positiva y baja.

\section{HIPÓTESIS ESPECÍFICA 2}

$\mathrm{H}_{0}$ : No existe relación entre el liderazgo y la planificación

\section{DEMOSTRACIÓN DE LAHIPÓTESIS}

Utilizamos el siguiente criterio:

Si la significancia asintótica $(p)>$ al nivel de significancia $(0,05)$, se acepta la $\mathrm{H}_{0}$. Si el valor de $p<0,05$ se rechaza $\mathrm{H}_{0}$.Aplicamos SPSS v25:

\section{Tabla 3.}

Relación entre el liderazgo y la planificación estratégica

\begin{tabular}{|c|c|c|c|c|}
\hline & & & $\begin{array}{l}\text { Planificacción } \\
\text { estratégica }\end{array}$ & Liderazgo \\
\hline \multirow{6}{*}{$\begin{array}{l}\text { Rho de } \\
\text { Spearman }\end{array}$} & & $\begin{array}{l}\text { Coeficiente de } \\
\text { correlación }\end{array}$ & 1,000 & $0,525^{\star *}$ \\
\hline & $\begin{array}{l}\text { Planificación } \\
\text { estratégica }\end{array}$ & Sig. (bilateral) & & 0,000 \\
\hline & & $\mathrm{N}$ & 86 & 86 \\
\hline & \multirow{3}{*}{ Liderazgo } & $\begin{array}{l}\text { Coeficiente de } \\
\text { correlación }\end{array}$ & $0,525^{\star *}$ & 1,000 \\
\hline & & Sig. (bilateral) & 0,000 & \\
\hline & & $\mathrm{N}$ & 86 & 86 \\
\hline
\end{tabular}

La correlación es significativa en el nivel 0,01 (Bilateral)

estratégica en la Facultad de Ciencias Empresariales, de la Universidad José Faustino Sánchez Carrión, 2019

$\mathrm{H}_{1}$ : Existe relación entre el liderazgo y la planificación estratégica en la Facultad de Ciencias Empresariales, de la Universidad José Faustino Sánchez Carrión, 2019

\section{DEMOSTRACIÓN DE LAHIPÓTESIS}

Utilizamos el siguiente criterio:

Si la significancia asintótica $(p)>$ al nivel de significancia $(0,05)$, se acepta la $\mathrm{H}_{0}$.

Si el valor de $p<0,05$ se rechaza $\mathrm{H}_{0}$.

Aplicamos SPSS v25: 


\section{Interpretación:}

En la tabla 3 se aprecia la significancia asintótica $(0,000)$ que es menor que el nivel de significación $(0,05)$, se rechaza la hipótesis nula y se acepta la hipótesis alterna (hipótesis del investigador).

Es decir, existe relación entre el liderazgo y la planificación estratégica en la Facultad de Ciencias Empresariales, de la Universidad José Faustino Sánchez Carrión, 2019. Además, la correlación de Rho de Spearman 0,525, de acuerdo a la escala de Bisquerra dicha correlación es positiva y baja.

\section{HIPÓTESIS ESPECÍFICA3}

$\mathrm{H}_{0}$ : No existe relación entre comunicación y planificación estratégica en la Facultad de Ciencias Empresariales, de la Universidad José Faustino Sánchez Carrión, 2019.

$\mathrm{H}_{1}$ : Existe relación entre comunicación y planificación estratégica en la Facultad de Ciencias Empresariales, de la Universidad José Faustino Sánchez Carrión, 2019.

\section{DEMOSTRACIÓN DE LAHIPÓTESIS}

Utilizamos el siguiente criterio:

Si la significancia asintótica $(p)>$ al nivel de significancia $(0,05)$, se acepta la $\mathrm{H}_{0}$.

Si el valor de $\mathrm{p}<0,05$ se rechaza $\mathrm{H}_{0}$.

Aplicamos SPSS v25:

\section{Tabla 4.}

Correlación entre comunicación y la planificación estratégica

\section{Correlaciones}

\begin{tabular}{|c|c|c|c|c|}
\hline & & & $\begin{array}{l}\text { Planificación } \\
\text { estratégica }\end{array}$ & Comunicción \\
\hline \multirow{6}{*}{$\begin{array}{l}\text { Rho de } \\
\text { Spearman }\end{array}$} & \multirow{3}{*}{$\begin{array}{l}\text { Planificación } \\
\text { estratégica }\end{array}$} & $\begin{array}{l}\text { Coeficiente de } \\
\text { correlación }\end{array}$ & 1,000 & $0,572^{* *}$ \\
\hline & & Sig. (bilateral) & & 0,000 \\
\hline & & $\mathrm{N}$ & 86 & 86 \\
\hline & \multirow{3}{*}{ Comunicación } & $\begin{array}{l}\text { Coeficiente de } \\
\text { correlación }\end{array}$ & $0,572^{* *}$ & 1,000 \\
\hline & & Sig. (bilateral) & 0,000 & \\
\hline & & $\mathrm{N}$ & 86 & 86 \\
\hline
\end{tabular}

La correlación es significativa en el nivel 0,01 (Bilateral)

\section{Interpretación:}

En tabla 4 se aprecia la significancia asintótica $(0,000)$ que es menor que el nivel de significación 0,05 , se rechaza la hipótesis nula y se acepta la hipótesis alterna (hipótesis del investigador). Es decir, existe relación entre comunicación y planificación estratégica en la Facultad de Ciencias Empresariales, de la Universidad José Faustino Sánchez Carrión, 2019. Además, la correlación de Rho de Spearman es 0,572, de acuerdo a la escala de Bisquerra dicha correlación es directa y moderada.

\section{HIPÓTESIS ESPECÍFICA4}

$\mathrm{H}_{0}$ : La motivación no se relaciona con la planificación estratégica en la Facultad de Ciencias Empresariales, de la Universidad José Faustino Sánchez Carrión, 2019

$\mathrm{H}_{1}$ : La motivación se relaciona con la planificación estratégica en la Facultad de Ciencias Empresariales, de la Universidad José Faustino Sánchez Carrión, 2019

\section{DEMOSTRACIÓN DE LAHIPÓTESIS}

Utilizamos el siguiente criterio:

Si la significancia asintótica $(p)>$ al nivel de significancia $(0,05)$, se acepta la $\mathrm{H}_{0}$.

Si el valor de $\mathrm{p}<0,05$ se rechaza $\mathrm{H}_{0}$.

Aplicamos SPSS v25: 
Tabla 4.

Correlación entre motivación y la planificación estratégica

Correlaciones

\begin{tabular}{|c|c|c|c|c|}
\hline & & & $\begin{array}{l}\text { Planificación } \\
\text { estratégica }\end{array}$ & Motivación \\
\hline \multirow{6}{*}{$\begin{array}{l}\text { Rho de } \\
\text { Spearman }\end{array}$} & & $\begin{array}{l}\text { Coeficiente de } \\
\text { correlación }\end{array}$ & 1,000 & $0,465^{\star *}$ \\
\hline & $\begin{array}{l}\text { Planificación } \\
\text { estratégica }\end{array}$ & Sig. (bilateral) & & 0,000 \\
\hline & & $\mathrm{N}$ & 86 & 86 \\
\hline & \multirow{3}{*}{ Motivación } & $\begin{array}{l}\text { Coeficiente de } \\
\text { correlación }\end{array}$ & $0,465^{* *}$ & 1,000 \\
\hline & & Sig. (bilateral) & 0,000 & \\
\hline & & $\mathrm{N}$ & 86 & 86 \\
\hline
\end{tabular}

La correlación es significativa en el nivel 0,01 (Bilateral)

\section{Interpretación:}

En tabla 5 se aprecia la significancia asintótica $(0,000)$ que es menor que el nivel de significación 0.05 , se rechaza la hipótesis nula y se acepta la hipótesis alterna (hipótesis del investigador). Es decir, La motivación se relaciona con la planificación estratégica en la Facultad de Ciencias Empresariales, de la Universidad José Faustino Sánchez Carrión, 2019. Además, la correlación de Rho de Spearman es 0.465 , de acuerdo a la escala de Bisquerra dicha correlación es positiva y baja.

\section{DISCUSIÓN}

En la presente investigación científica se realizó una comparación de los resultados obtenidos con otras investigaciones similares, distinguiendo las variables estudiadas o su respectiva relación, destacando aspectos de similitud o discrepancia con los antecedentes y fuentes teóricas citadas en la presente investigación.

Los resultados obtenidos en esta investigación conducen en términos generales a establecer que existe relación entre el trabajo en equipo y la planificación estratégica en la Facultad de Ciencias Empresariales, de la Universidad José Faustino Sánchez Carrión, 2019. Además, la correlación de Rho de Spearman es 0,605, de acuerdo a la escala de Bisquerra dicha correlación es positiva y moderada.

Este estudio tiene similitud con la investigación de Sullca, (2018). En su tesis Gestión del cambio organizacional y planificación estratégica en el Programa de Complementación Alimentaria. Municipalidad de SJ. L, 2018". Tesis para optar el grado académico de: Maestra en Gestión Pública, en la Escuela de Postgrado Universidad Cesar Vallejo PERÚ. Logró demostrar que hay una relación de la variable gestión del cambio organizacional y planificación estratégica de los trabajadores del Programa de Complementación Alimentaria- Municipalidad de S.J.L, 2018, hallándose un coeficiente de correlación Rho Spearman de $0,837^{* *}$ y nivel de significancia 0,00 lo que significó un nivel de relación alta entre las variables.

En forma similar también con los aportes de Zapana, (2017). En su tesis "Grado de relación entre la planificación estratégica y la toma de decisiones en las instituciones educativas secundarias públicas de la Ugel Chucuito - julio - 2016". La cual concluye: que la planificación estratégica y el empowerment influyen significativamente en el clima organizacional de los docentes de la Institución Educativa "Juana Alarco de Dammert”, Miraflores, Lima, 2015. (Con un $p<0,05$ y Rho de Spearman $=0,982$ correlación positiva fuerte). El resultado obtenido en la presente investigación guarda relación con el autor mencionado, es decir existe asociación entre la dinámica de equipos y la planificación estratégica en la Facultad de Ciencias Empresariales, de la Universidad José Faustino Sánchez Carrión, 2019. Además, la correlación de Rho de Spearman es 0.393 , de acuerdo a la escala de Bisquerra dicha correlación es positiva y baja.

Así mismo Diaz y Prialé (2018). En su tesis “"La Planificación Estratégica como herramienta de mejora en la gestión del gobierno Municipal de la Molina" para optar el grado académico de Maestro en Gestión Pública en la Universidad Peruana de Ciencias Aplicadas Escuela de Postgrado. Logro determinar que Existe evidencia que el nivel de cumplimiento de la misión de la planificación estratégica es una herramienta de mejora que se relaciona significativamente con la gestión del gobierno municipal de La Molina.

Estos resultados tienen similitud con lo hallado en la presente investigación, es decir, existe relación entre el liderazgo y la planificación estratégica en la Facultad de 
Ciencias Empresariales, de la Universidad José Faustino Sánchez Carrión, 2019. Además, la correlación de Rho de Spearman 0,525, de acuerdo a la escala de Bisquerra dicha correlación es positiva y baja.

En forma similar también con los aportes de Mella, (2018). En su tesis "Desafíos del liderazgo, a través de la gestión de la planificación estratégica, caso CREAVI Spa". Demostró que La implementación de un plan estratégico, como herramienta, se plantea con pocos indicadores inicialmente o considerar aquellos más específicos y necesarios, reforzando los puntos de las personas y procesos.

El desarrollo de una adecuada planificación, mantiene una relación entre los objetivos y recursos de la organización, además de obtener un mejor partido de las oportunidades que se presentan en el mercado. Con los lineamientos de la estrategia, son la base fundamental para alcanzar las metas, siempre integrando a todos sus actores y se espera que sean internalizados en sus directivos y equipo de trabajo, obteniendo como resultado un personal altamente motivado por los beneficios que ello acarrea. El resultado obtenido en la presente investigación guarda relación con el autor mencionado, es decir existe relación entre comunicación y planificación estratégica en la Facultad de Ciencias Empresariales, de la Universidad José Faustino Sánchez Carrión, 2019. Además, la correlación de Rho de Spearman es 0,572, de acuerdo a la escala de Bisquerra dicha correlación es directa y moderada.

Así mismo con los aportes de Ortiz, (2015). En su tesis "Cómo manejar la comunicación interna en un proceso de planeación estratégica. Caso: Ministerio de Turismo" Investigación de Maestría en Desarrollo del Talento Humano presentado en la Universidad Andina Simón Bolívar Sede Ecuador. Logro determinar que La planeación estratégica en el MINTUR, destinada al logro de sus objetivos estratégicos, se maneja bajo un Plan Anual, sobre el que se realiza una programación mensual a partir de los logros y avances alcanzados y en la que la comunicación interna se hace imprescindible pero que se realiza sin aprovechar los beneficios y características de los canales de comunicación de la institución.

Estos resultados tienen similitud con lo hallado en la presente investigación, es decir, La motivación se relaciona con la planificación estratégica en la Facultad de Ciencias Empresariales, de la Universidad José Faustino Sánchez Carrión, 2019. Además, la correlación de Rho de Spearman es 0.465 , de acuerdo a la escala de Bisquerra dicha correlación es positiva y baja.

\section{CONCLUSIONES}

De las contrastaciones de hipótesis concluyo en:

Se logró demostrar que existe relación entre el trabajo en equipo y la planificación estratégica en la Facultad de Ciencias Empresariales, de la Universidad José Faustino Sánchez Carrión, 2019. Con significancia asintótica de $(0,000)$ y con coeficiente de correlación de Rho de
Spearman $(r=0,605)$. Es decir, dicha correlación es positiva directa y moderada. Este resulta indica que existe moderada trabajo en equipo y por ello también la planificación estratégica es moderada.

Logré determinar existe asociación entre la dinámica de equipos y la planificación estratégica en la Facultad de Ciencias Empresariales, de la Universidad José Faustino Sánchez Carrión, 2019. Con nivel de significancia de $(0,000)$ y con coeficiente de correlación de Rho de Spearman ( $r=0,393)$, de acuerdo a la escala de Bisquerra dicha correlación es positiva y baja. Es decir, en la Facultad de Ciencias Empresariales los docentes y administrativos contribuyen moderadamente en la distribución de las actividades académicas y administrativas, razón por el cual lo planificado se cumple también moderadamente.

Se demostró estadísticamente que existe relación entre el liderazgo y la planificación estratégica en la Facultad de Ciencias Empresariales, de la Universidad José Faustino Sánchez Carrión, 2019. Con nivel de significancia de $(0,000)$ y con coeficiente de correlación de Rho de Spearman $(r=0,525)$, de acuerdo a la escala de Bisquerra dicha correlación es positiva y baja. Es decir, los docentes y trabajadores administrativos muestran su liderazgo moderadamente en los diversos eventos académicos y administrativos que organiza la Facultad de Ciencias Empresariales.

Se demostró estadísticamente que existe relación entre la comunicación y planificación estratégica en la Facultad de Ciencias Empresariales, de la Universidad José Faustino Sánchez Carrión, 2019. Con nivel de significancia del $(0,000)$ y con coeficiente de correlación de Rho de Spearman ( $r=0,572)$, de acuerdo a la escala de Bisquerra dicha correlación es positiva y baja. Es decir, en la facultad de Ciencias Empresariales los docentes y administrativos fomentan la empatía, comunicación moderadamente entre sus estudiantes y colegas. Por ello se cumple pocas veces la planificación estratégica de la facultad.

Además, logré demostrar que la motivación se relaciona con la planificación estratégica en la Facultad de Ciencias Empresariales, de la Universidad José Faustino Sánchez Carrión, 2019. Con nivel de significancia del $(0,000)$ y con coeficiente de correlación de Rho de Spearman $(r=0,465)$, de acuerdo a la escala de Bisquerra dicha correlación es positiva y baja. Es decir, los docentes muestran poca motivación en su labor pedagógico y administrativo, de la misma manera el trabajador administrativo muestra poca motivación en el momento de atender a los estudiantes. Por ello los objetivos institucionales de la Facultad se cumplen moderadamente. 


\section{REFERENCIAS}

Ascencio, A., Campo, J., Ramírez, A., \& Zapata, L. (2016). Importancia de la planeación estratégica en las áreas de gestión humana de las organizaciones. Medellín Colombia: Revista Fundación Universitaria Luis Amigó.

Barreda, H. (2016). PLANEAMIENTO ESTRATEGICO EN UNIVERSIDADES DEDE AMERICA LATINA. Revista GUAL, Florianópolis, 257277.

Chiavenato, I. (2009). COMPORTAMIENTO ORGANIZACIONAL La dinámica del éxito en las organizaciones (Segunda ed.). México: McGRAWHILL/INTERAMERICANAEDITORES, S.A. de C.V.

Correa, H. (2015). PLANIFICACIÓN ESTRATÉGICA PARA EI DESARROLLO REGIONAL Y RURAL (Vol. Consorcio de Investigacón económica y Social (CIES). ). Lima: Universidad Nacional de Piura.

Díaz, G., Manco, C., \& Prialé, P. (2018). La Planificación Estratégica como herramienta de mejora en la gestión del gobierno Municipal de la Molina. Tesis para optar el grado académico de Maestro en Gestión Pública: Universidad Peruana de Ciencias Aplicadas Escuela de Postgrado.

Fundacion Universitaria SEDUCA-FUNLAM. (2008). EL TRABAJO EN EQUIPO. Antioquia, Colombia: Proyecto Formación de Directivos Docentes en Antioquia.

Guardia, C. (2014). Contextualización y conceptualización de la planificación estratégica de la comunicación de marca aplicada al Internal Branding: propuesta de un modelo teórico". (Tesis Doctoral): UniversitatAutònoma de Barcelona .
Koontz, H., Weihrich, H., \& Cannice, M. (2012). Administración una perspectiva global y empresarial (decimocuarta edición ed.). México: McGRAWHILL/INTERAMERICANAEDITORES S.A. DE C.V.

Mella, Y. (2018). Desafíos del liderazgo, a través de la gestión de la planificación estratégica, caso CREAVI Spa. (Tesis MBA, Magíster en Gestión Empresarial): Universidad Técnica Federico Santa María, Chile .

Ortiz, N. (2015). Cómo manejar la comunicación interna en un proceso de planeación estratégica. Caso: Ministerio de Turismo . (Tesis de Maestría en Desarrollo del Talento Humano): Universidad Andina Simón Bolívar Sede Ecuador.

Robbins, S., \& Judge, T. (2009). Comportamiento organizacional. (Decimotercera edición ed.). México: PEARSONEDUCACIÓN.

Stanton, W., Etzel, M., \& Walker, B. (2007). Fundamentos de MARKETING ((Decimocuarta edición) ed.). México: McGRAW-HILL/INTERAMERICANA EDITORES, S.A. DE C.V.

Sullca, G. (2018). Gestión del cambio organizacional y planificación estratégica en el Programa de Complementación Alimentaria. Municipalidad de SJ.L, 2018. (Tesis de Maestria en Gestión Pública): Escuela de Postgrado Universidad Cesar Vallejo - Perú.

Universidad Católica Sedes Sapientiae. (2011). Instrumentos de planificación en los gobiernos locales del Perú: propuesta para sistematización de la planificación local. Los Olivos, Lima-Perú: Talleres gráficos de Llamkay S.A.C. 